\title{
Diagnostic challenges in childhood sarcoidosis
}

Linda Manaa ${ }^{1}$, Monia Youssef ${ }^{1}$, Chokri Chouchene ${ }^{1}$, Nouha Benabdejlil ${ }^{1}$, hichem hajali ${ }^{1}$, Sleh chouchene ${ }^{1}$, and Jameleddine Zili ${ }^{1}$

${ }^{1}$ Fattouma Bourguiba University Hospital of Monastir

January 18,2022

\begin{abstract}
The aim of our clinical image is to emphasize the value of a careful skin examination in the diagnosis of early-onset sarcoidosis in children.
\end{abstract}

Title page

Type of the article: image

Title : Diagnostic challenges in childhood sarcoidosis

Authors names: Manaa $\mathrm{L}^{1}$, Youssef $\mathrm{M}^{1}$, Chouchène $\mathrm{C}^{2}$, Ben abdejlil $\mathrm{N}^{3}$, Belhadjali $\mathrm{H}^{1}$, Chouchène $\mathrm{S}^{2}$, Zili $\mathrm{J}^{1}$

Affiliations: ${ }^{1}$ Dermatology Department, Fattouma Bourguiba University Hospital, University of Monastir, TUNIISIA

${ }^{2}$ Pediatric Department, Fattouma Bourguiba University Hospital, University of Monastir, TUNIISIA

${ }^{3}$ Anatomopathology Department, Fattouma Bourguiba University Hospital, University of Monastir, TUNIISIA

Corresponding author: Manaa Linda; MD,

address: Dermatology Department, Fattouma Bourguiba University Hospital, University of Monastir, 5000 Monastir, TUNIISIA

Email address:lindamanaa6@gmail.com; Telephone number: 0021629423218

Manuscript count: 212

Figures: 02, 0 Table

Funding : None, The authors have received no funding from any agency in the public, private, or not-forprofit sectors.

Conflict of interest: The authors declare that there are no conflicts of interest in this work.

\section{Acknowledgements:}

The patient' parents in this manuscript have given written informed consent to the publication of the case details of their child.

Abstract : 
The aim of our clinical image is to emphasize the value of a careful skin examination in the diagnosis of early-onset sarcoidosis in children.

\section{Case presentation :}

A 2-year-old child had a one year history of polyarthritis and recurrent rash that was not objectified during consultations. The diagnosis of juvenile idiopathic arthritis was made. The patient was treated with nonsteroidal anti-inflammatory drugs and methotrexate. He was hospitalized after 2 months because of severe arhralgia and functional impairment. On skin examination, he had flesh-colored, non-itchy, $4 \mathrm{~mm}$ papular lesions on the arms and forearms with micropapular lesions on the trunk giving a keratosis pilaris-like appearance (figure 1 ). The ophthalmologic examination showed anterior uveitis with Koeppe and Busacca nodules. The child did not have respiratory symptoms or lymphadenopathy. The chest x-ray and blood tests were without abnormalities. A skin biopsy showed non-necrotizing granulomatous inflammation of the dermis, confirming the diagnosis of sarcoidosis (figure $\mathbf{2}$ ). The patient was treated with systemic corticosteroids with improvement of clinical signs.

Sarcoidosis in children poses a diagnostic challenge, especially before the age of 5 , given the absence of the classic signs of sarcoidosis. ${ }^{1}$ It is characterized at an early age by the triad: uveitis, arthritis and rash. The cutaneous manifestations are variable: discrete and confluent papules, eczematiform, ichthyosiform lesions, subcutaneous nodules, symmetrical maculopapular lesions or erythroderma. ${ }^{2}$ The rash is characterized by exacerbations and remissions. It often precedes other signs and can be discrete and go unnoticed.

Authorship: All authors had access to the data and a role in writing this manuscript.

\section{Author contributions :}

The authors fulfill the ICMJE Criteria for Authorship and contributed equally.

Dr Manaa Linda, is the guarantor of the content of the manusript, included the data and analysis. Dr Youssef Monia contributed to interpretation of data and revision of the manuscript. Dr Chouchene Chokri contributed to data collection. Dr Ben abdejlil Nouha and Belhadjali hichem contributed to analysis and interpretation of data, revised it critically. Dr zili jamaleddine and Dr Chouchene Sleh contributed to final approval of the version of the manuscript to be submitted.

\section{Consent :}

The patient' parents in this manuscript have given written informed consent to the publication of the case details of their child.

\section{References:}

1. Lee JY, Yun SJ, Hong JS, Lee AY, Lee SH. Early-onset sarcoidosis presenting as a generalized papular eruption. J Dermatol [Internet]. 2019 [cite 11 janv 2022];46(12). Disponible sur: https://onlinelibrary.wiley.com/doi/10.1111/1346-8138.15068

2. Agarwal K, Barua S, Adhicari P, Das S, Marak R. Early-onset sarcoidosis and juvenile idiopathic arthritis:A diagnostic dilemma. Indian J Dermatol Venereol Leprol. 2016;82(5):542.

\section{Figure legends:}

Figure 1: Flesh-colored, non-itchy, papular lesions on the arms and forearms.

Figure 2: Dermal and subcutaneous non-necrotizing granulomas composed of epithelioid histiocytes with sparse lymphocytic infiltrate (H\&E x 100). 

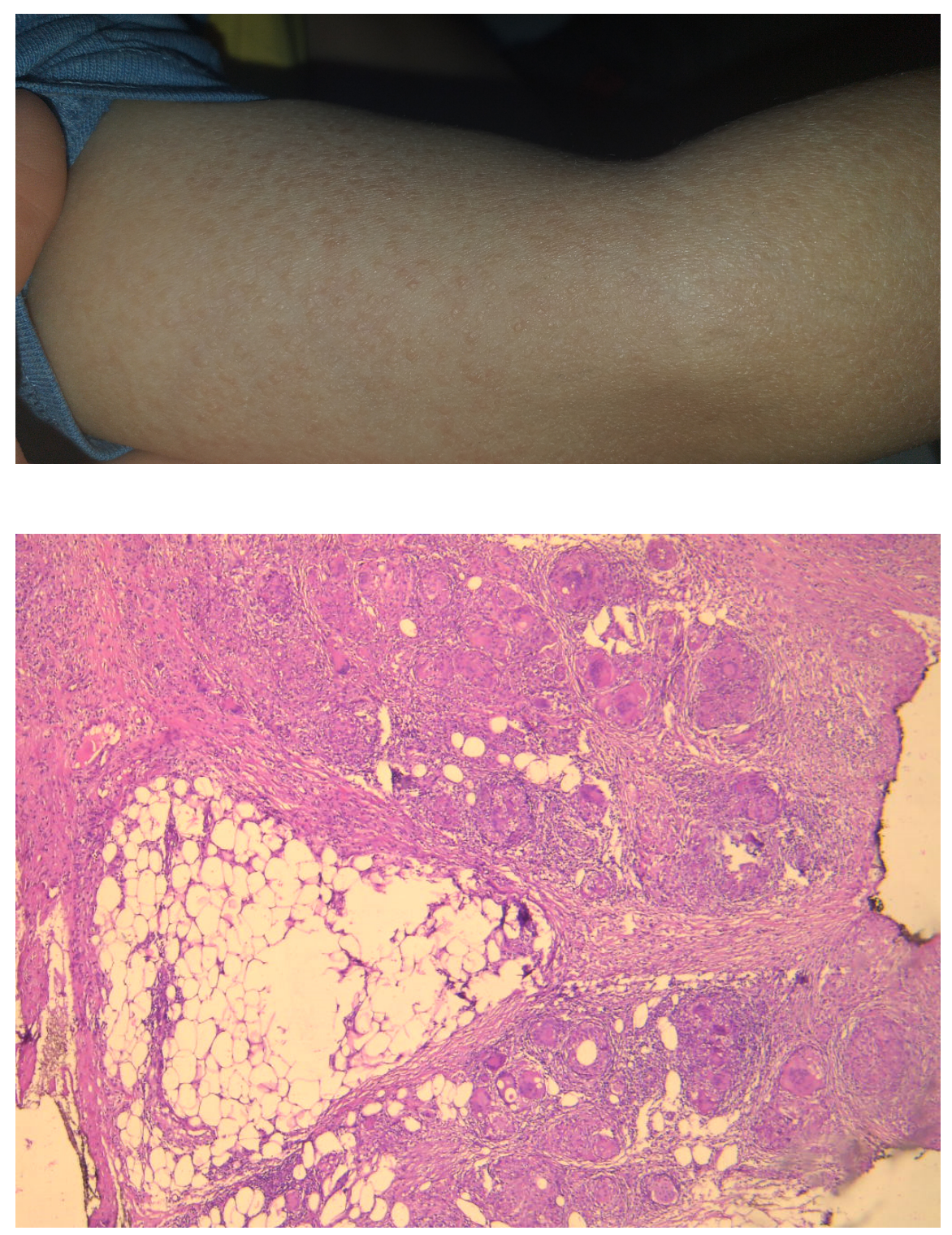